\title{
Levels of Depression and Anxiety Post-Mastectomy in Breast Cancer Patients at a Public Sector Hospital in Karachi
}

\author{
Sara Khan ${ }^{1 *}$, Naveed Ali Khan², Ata Ur Rehman², Iqra Khan², Khursheed A \\ Samo $^{2}$, Amjad Siraj Memon ${ }^{2}$
}

\begin{abstract}
Background: There is a noticeable change in the approach with which women nowadays seek help for diseases like breast cancer, primarily due to awareness campaigns, but what happens after surgical procedures is of great significance too. This study focused on the several psychological connotations attached to mastectomy and how the patients cope. Objective: To understand the pattern of anxiety and level of depression among Pakistani patients undergoing mastectomy. Materials and Methods: The sample size consisted of 88 patients who had undergone mastectomy at the different surgical units of the Civil Hospital, Karachi, from January 2012- December 2014; The questionnaire was administered before they were discharged i.e. within 3 days of surgical procedures. The patients were asked if they were willing to participate in this study, those who agreed signed the consent form and then we preceded by asking questions with a standardized tool. A self made questionnaire was constructed keeping in mind the nature and specification of the disease, which consisted of 20 questions related to anxiety and depression, focusing on a mixture of psychological and physiological symptoms. Results: There were a total of 88 patients out of which $36(41 \%)$ were aged 51 to 60 years, $24(27.2 \%)$ of the patients were in the age category of 41 to 50 years, 17 aged 61 and above $(19.3 \%)$ and only $11(12.5 \%)$ 30-40 years of age, the youngest of all the age categories. The pattern of depression and anxiety was found to be similar among all age categories, severe depression and anxiety predominating over moderate level of such symptoms, with only relatively few patients sufering mild or no depression symptoms. Conclusions: It was concluded that going through mastectomy leads to moderate to severe levels of depression and anxiety, primarily because the females feel incomplete and insecure after losing a part of themselves.
\end{abstract}

Keywords: Mastectomy - breast cancer - depression - anxiety - Pakistani females

Asian Pac J Cancer Prev, 17 (3), 1337-1340

\section{Introduction}

A mastectomy is surgery to remove a breast or part of a breast. It is usually done to treat breast cancer (Parkin et al., 2005).

Types of breast surgery include; (simple) mastectomy i.e. removal of breast tissue and nipple whereas modified radical mastectomy is removal of the breast, most of the lymph nodes under the arm, and often the lining over the chest muscles; another type of breast surgery is lumpectomy, which is a surgery to remove the tumor and a small amount of normal tissue around it (Iwamitsu et al., 2005).

Anxiousness is a human phenomenon, its universal. Normally anxiety includes a state of helplessness. The physiological symptoms include fast breathing, heart palpitation, loss of appetite, insomnia, sweating, restlessness, shortness of breath and nausea (Beck and Steer, 1993).
Depression is common among patients of terminal illnesses. If we look at the survey reports, it is state that $50 \%$ of the patients of cancer report symptoms which lie under the jurisdiction of the depression and $20 \%$ have definitive depressive illness (Barraclough, 1999).

There are strong linkages found between emotion and cancer, it effects the growth of cancer cell by weakening the natural defenses of the body whereas relaxed and hopeful state makes these defenses sounder for the overall working of the body and increases immunity (Myers, 2004).

It is commonly observed that people prone to depression and anxiety or who have a genetic disposition to such mental illnesses tend to be at higher risk for terminal illnesses as their habit of worrying every time leads to a weakened immune system, in result making them vulnerable to such diseases (Coleman and Broen, 1972)

According to Dr. Aron Beck's paradigm of cognitive

${ }^{1}$ Clinical Psychology, The Indus Hospital, Karachi, ${ }^{2}$ Surgical Unit 3, Civil Hospital Karachi, DUHS *For correspondence: dr_ata_rehman@hotmail.com 
behavior; depressive symptoms are a product of negative thoughts and dysfunctional beliefs hence it can be concluded that the more negative thoughts one experiences, the more depressed one will be, these are those negative anticipations which leads to the deterioration of the personality, cognitions and as a result eventually leads to negative behavioral changes (Gilmer, 1975).

Beck also asserts that there are three main dysfunctional belief themes (or "schemas") that dominate depressed people's thinking: 1) I am defective or inadequate, 2) All of my experiences result in defeats or failures, and 3) The future is hopeless. Together, these three themes are described as the Negative Cognitive Triad. When these beliefs are present in someone's cognition, depression is very likely to occur (if it has not already occurred) (Beck, 1967).

A research study done on Taiwanese 203 hospitalized cancer patients, which assessed their depression and anxiety level by using (HADS) hospital anxiety and depression scale. The cancer patient's anxiety can be predicted by their functional status and effect of their treatment. It was found to be a valid argument that depression and anxiety has its repercussions attached to the patients on the course of treatment for cancer (Chen et al., 2000).

When satisfaction level of women after mastectomy was assessed in a research, it was found that some women had expressed dissatisfaction due to postoperative complications. The patients were assessed about their overall satisfaction such as their physical and emotional recovery, the support of their family especially husband and the side effects of the surgery (Borgen et al., 1998).

The diagnosis of cancer is usually associated with fear of pain, death, and changes in sexual life, body image, abandonment, ability to work, financial issues and relationships, which eventually leads to depression and anxious state (William, 2002).

Several reports suggest that women undergoes profound psychosocial distress during their treatment, however the level of depression varies from women to women, it depends on their individual ability to react to the circumstances in a more conducive way rather than complaining about the events and situations which are inevitable (Massie and Holland, 2001).

It can be inferred from the above mentioned studies that depression and anxiety are prevalent among Pakistani female patients, but less work is done on this subject matter. This research study aims to promote and highlight knowledge about depression and anxiety among patients post mastectomy.

\section{Materials and Methods}

The sample size consisted of 88 in patients at the different surgical units of the Civil hospital, Karachi, from January 2012- December 2014; who had under gone mastectomy. The induction criterion was any state that required mastectomy. The questionnaire was administered before they were discharged i.e. within 3 days of the surgical procedure. The patients were asked if they were willing to participate in this study, those who agreed signed the consent form and then it was preceded by asking them questions from a standardized tool. A self made questionnaire was constructed keeping in mind the nature and specification of the disease, which consisted of 20 questions related to anxiety and depression, a mixture of psychological and physiological symptoms attached to it keeping in mind the nature of the disease.

The cut off score is 12 .i.e. first 12 questions measure psychological symptoms whereas the rest measures physiological symptoms. There are four modes of answer i.e. never, sometimes, often and always designated 0,1 , 2 and 3 respectively.

The total score ranging from 0-16, signify no depression and anxiety symptoms, 17- 32 signifies mild depression and anxiety symptoms, 33-48 signifies moderate symptoms of depression and anxiety whereas 49 and onwards identifies that the patient is severely under depression and is highly anxious i.e. the patients who scored less than $80 \%$ of the minimum total questions $16 / 20$ are not depressed and anxious and vice versa.

The consent form consisted of the title and objective of the study along with the basic demographics of the patients such as age, socio economic status, qualification, ethnicity, mother tongue and occupation. The patients were asked to give thumb impression or sign the consent form, if they agree to participate in the research study.

\section{Results}

As the Table 1 shows that there were a total of 88 patients out of which $36(41 \%)$ were aged [ 51 to 60 ] years, $24(27.2 \%)$ of the patients lies under age category of [41 to 50] years, age category of [61 and above] were 17 in number $(19.3 \%)$ whereas the least number of patients i.e. $11(12.5 \%)$ comprised of [30-40] years of age, which happens to be the youngest of all the age categories as shown in Figure 1.

As table 2 depicts that irrespective of the age categories, the severity of anxiety and depression was found in $46 / 88$ patients i.e. $52.3 \%$, whereas moderate symptoms of anxiety and depression was found in (27/88) patients .i.e. $30.7 \%$. Another noticeable fact found through this study was that only $(4 / 88)$ patients i.e. $4.5 \%$ of the total sample didn't report symptoms of anxiety and depression, whereas only (11/88) i.e. $12.5 \%$ reported mild anxiety and depression as shown in figure 2 .

If we keep the age categories in mind, we can easily infer that in category [30-40 years], severe depression and anxiety symptoms were felt by $54.5 \%$ (6/11) patients; $27.3 \%(3 / 11)$ felt moderate level of depression and

Table 1. Total Number and Percentages of Patients in Each age Category

\begin{tabular}{lcc}
\hline Age & $\begin{array}{c}\text { Number of patients } \\
(\mathrm{n} \text { size })\end{array}$ & $\begin{array}{c}\text { Percentage } \\
(\%)\end{array}$ \\
\hline $30-40$ years & 11 & 12.5 \\
$41-50$ years & 24 & 27.2 \\
$51-60$ years & 36 & 41 \\
$61-$ above & 17 & 19.3 \\
\hline Total & 88 & 100 \\
\hline
\end{tabular}


Table 2. Levels Of Depression and Anxiety among Different age Categories

\begin{tabular}{|c|c|c|c|c|c|c|c|c|c|c|}
\hline \multirow[t]{2}{*}{ Age of patient } & \multicolumn{2}{|c|}{$\begin{array}{l}\text { No Anxiety } \\
\text { \& Depression }\end{array}$} & \multicolumn{2}{|c|}{$\begin{array}{l}\text { Mild Anxiety } \\
\text { \& Depression }\end{array}$} & \multicolumn{2}{|c|}{$\begin{array}{l}\text { Moderate Anxiety } \\
\text { \& Depression }\end{array}$} & \multicolumn{2}{|c|}{$\begin{array}{l}\text { Severe Anxiety \& } \\
\text { Depression }\end{array}$} & \multicolumn{2}{|c|}{ Total } \\
\hline & $\mathrm{N}$ size & $\%$ & $\mathrm{~N}$ size & $\%$ & $\mathrm{~N}$ size & $\%$ & $\mathrm{~N}$ size & $\%$ & $\mathrm{~N}$ size & $\%$ \\
\hline $30-40$ years & 0 & 0 & 2 & 18.2 & 3 & 27.3 & 6 & 54.5 & 11 & 100 \\
\hline $41-50$ years & 1 & 4.2 & 4 & 16.6 & 7 & 29.2 & 12 & 50 & 24 & 100 \\
\hline $51-60$ years & 1 & 3.8 & 3 & 8.3 & 11 & 30.5 & 21 & 58.3 & 36 & 100 \\
\hline 61 - above & 2 & 11.7 & 2 & 11.7 & 6 & 35.3 & 7 & 41.3 & 17 & 100 \\
\hline Total & \multicolumn{2}{|c|}{$4(4.5 \%)$} & \multicolumn{2}{|c|}{$11(12.5 \%)$} & \multicolumn{2}{|c|}{$27(30.7 \%)$} & \multicolumn{2}{|c|}{$46(52.3 \%)$} & \multicolumn{2}{|c|}{$88(100 \%)$} \\
\hline
\end{tabular}

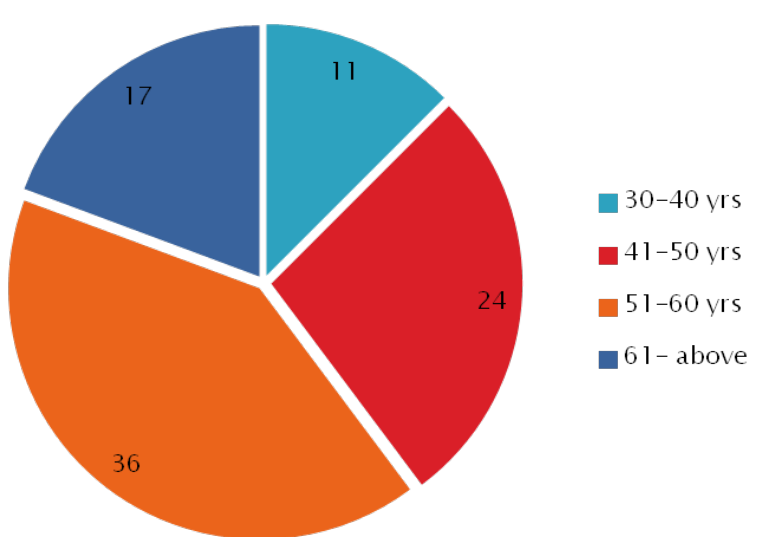

Figure 1. Total Number of Patients in Each age Category

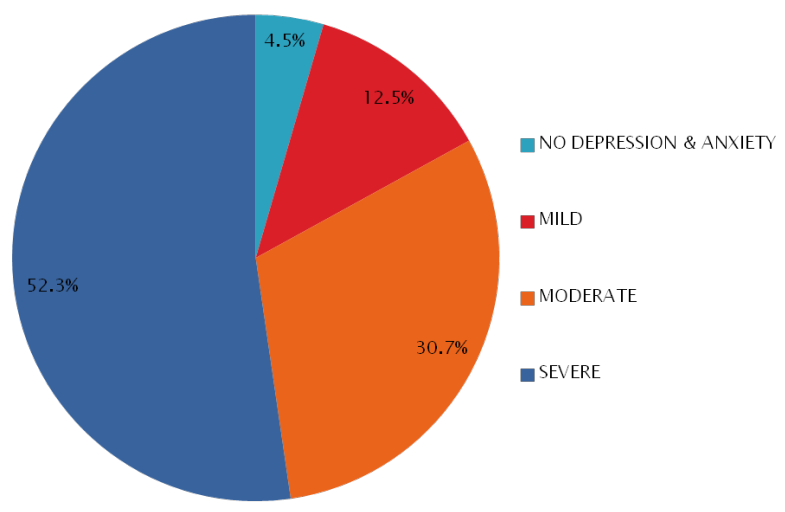

Figure 2. Percentages of Level of Depression in Mestectomy Patients

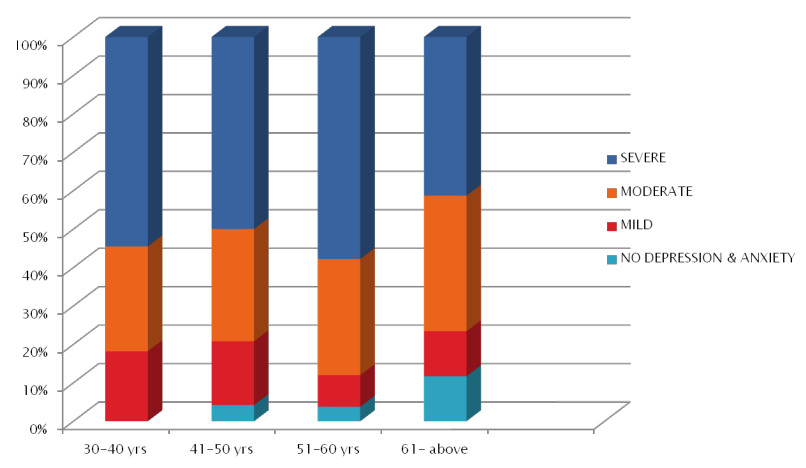

Figure 3. Percentages of level of Depression \& anxiety among age categories

anxiety whereas $18.2 \%(2 / 11)$ felt mild level of anxiety and depression.

In age category [41-50 years], 50\% (12/24) felt severely depressed and anxious, 29.2\% (7/24) felt moderately anxious and depressed; whereas $16.6 \%$ (4/24) and $402 \%(1 / 24)$ felt mild or no depression symptoms respectively.

In age category [51-60 years] 58.3\% (21/36) felt severely depressed and anxious, $30.5 \%$ (11/36) felt moderately depressed and anxious, whereas $8.3 \%$ (3/36) and $2.9 \%(1 / 36)$ felt mild or no depression symptoms respectively.

And in category [61- above years], severe depression and anxiety symptoms were felt by $41.3 \%$ (7/17) patients; $35.3 \%(6 / 17)$ felt moderate level of depression and anxiety whereas $11.7 \%(2 / 17)$ felt mild level of anxiety and depression and/ or no depression and anxiety at all, as shown in figure 3 .

\section{Discussion}

As the result findings suggest that the highest number of enrollments during the two years of research's data collection in public sector hospital was of the patients bearing age between 51 to 60 years. This age bracket comes under the peripheries of adulthood and this is the time when most women in Pakistan undergoes menopausal symptoms, it's indeed a transitional phase for most women as they go through physiological and psychological changes during this period.

If we broaden the age bracket we can see that the second largest number of enrolments fall under the age category of 41 to 50 years hence, we can easily infer that from 41 to 60 years of age is the threshold of the most prevalent occurrence or reporting of the disease by the patients.

Usually it is seen that this is the age when women start getting conscious and cautious of their health related issues primarily due to the changes they feel due to menopausal symptoms which start to occur and also because they feel it's time to maintain their health as they are getting older.

The least reported cases were from the age bracket 3040 years, which is the youngest of all the age categories, if we have a closer look at this situation from a social angle, women of this age group are relatively younger , hence they feel they are safe from any such cancer or tumor, which is primarily due to lack of awareness, another facet is that this is the age of utmost busyness for women , as they are newly married or new mothers by then and have enough workload on their shoulders to give precise attention to such symptoms.

Another social angle is of shyness; women in Pakistan are hesitant to discuss or share such issues especially related to their body, and hence they resort to female 
general practitioners when the situation is out of their control.

It is observed that majority of women relate breasts with women hood, hence any scar or radical excision of this organ makes them incomplete from the aspect of being a women, which has immense psychological repercussions.

The reaction of their husbands, how they will react to such a terminal illness, surgical procedure and after care regimen is also a reason for such high levels of depression and anxiety in patients. They feel themselves as burden on their better halves and fear the repercussions in the longer run.

A whole skeptical approach creates a vicious circle, hence the negative thinking pattern such as life has come to an end, I m useless etc; becomes the most prevalent thoughts leading to hopelessness and pessimism in patients of mastectomy.

According to Beck depressive's school of thoughts symptoms are a product of negative thoughts and dysfunctional beliefs hence it can be concluded that the more negative thoughts you experience, the more depressed you will be (Pasquini and Biondi, 2007).

Several reports suggest that women undergoes profound psychosocial distress during their treatment, however the level of depression varies from women to women (Zabalegui et al., 2005).

This high level of depression and anxiety scores depict insecurity related to their future lives and has social, emotional, psychological connections attached to it. Such patients show withdrawal symptoms such disinterest in routine life.

Only $4.5 \%$ of the sample population reported to have no depression and anxiety, which is relatively a smaller number, the main reason behind this was found to be social support, i.e. positive regard from the family members especially husband, if married, basic knowhow of the surgical procedure, awareness and the hope that they will get rid of this disease once and for all after the mastectomy and their lives will be back to normal again, free of pain and stigma.

Two studies conducted in France and Australia included samples of women who had been seeking appointments of cancer genetic clinics (Meiser et al., 2000).

Unfortunately, this is a disease that is not only stigmatized in our society but its genetic disposition makes the patients more fearful towards the younger women in their family which results in making them more depressed and anxious even after the surgical procedure.

A research finding done between 1960 and 1993 concludes that breast cancer can be reduced among the women who had a family history of the disease were identified and re contacted as they at a greater risk of it (Hartmann et al., 1999).

In conclusion, It was concluded that going through mastectomy leads to moderate to severe level of depression and anxiety, primarily because the females feel incomplete and insecure after losing a part of themselves. There are lots of psychological and social connotations attached to it. This insecurity is not just prevalent among young females but also in old aged females too, they hesitate to discuss it and feel incomplete with their missing part, some associate it with beauty dynamics while others feel that they were used to it. Social approval and also that of their husbands, if married; also lays a matter of concern for the patients of mastectomy.

Recommendations: It is highly recommended to recruit psychologists and mental health practitioners to facilitate a smooth process of mastectomy or any surgical procedure at large. Pre and post operative psychological analysis should be mandatory, just as basic medical work up. As breast surgeries are unfortunately taken as a matter of shame by most of the patients and also their families too, hence female staff can prove to be more facilitative in this regard. Breast cancer awareness programs should work hand in hand for the rehabilitation of the patients of mastectomy. One day training or awareness workshops for such patients before and after the surgery can also do wonders in dropping down their depression and anxiety levels.

\section{References}

ABarraclough J (1999). Cancer and Emotion.In 'A practical guide to psycho oncology',3rd Eds. John Wiley \& Sons, London pp 104-124 Numbers 222-3.

Beck A and Steer R (1993). Beck Anxiety Inventory.The Psychological Cooperation and ISP logo, New York pp 4-20 Numbers 20-3.

Beck AT (1967). Depression. IN 'Clinical, Experimental and Theoretical Aspects'. University of Pennsylvania press, philadelphia pp 99-289 Numbers 19104-9.

Borgen I, Hill D, Tran N, et al (1998). Patient regrets after bilateral prophylactic mastectomy. Ann Surg Oncol, 5. 603-6.

Chen, Ling M, Kun H, Hsing C (2000). Anxiety and depression in Taiwanese cancer patients with and without pain. J Advanced Nursing, 4, 944-951.

Coleman J, Broen WE (1972). Abnormal Psychology and Modern Life, $4 \mathrm{r}^{\text {th }}$ Eds. Scott, Foresman and Company, Michigan pp 425-621 Numbers 798.

Gilmer B (1975). Adjustment in Living and Work. In 'Applied Psychology', 2nd Eds. Mc Graw Hill Publishing Company Ltd, New Delhi pp 305-411 Numbers 470-9.

Hartmann LC, Schaid DJ, Woods JE, et al (1999). Efficacy of bilateral prophylactic mastectomy in women with a family history of breast cancer. $N$ Engl J Med, 340, 77-84.

Iwamitsu Y, Shimoda K, Abe H, et al (2005). Anxiety, Emotional Suppression, and Psychological Distress Before and After Breast Cancer Diagnosis. Psychosomatics, 46, 19-24.

Massie J and Holland C (2001). The cancer patients with pain. Psyc Complications Their Management, 71, 243-247.

Meiser B, Butow P, Friedlander M,et al (2000). Intention to undergo prophylactic bilateral mastectomy in women at increased risk of developing hereditary breast cancer. J Clin Oncol, 18, 2250-7.

Myers D (2004). Psychology in modules, $7^{\text {th }}$ Eds. Worth publishers, New York pp 567-705 Numbers 752-4.

Parkin M, Bray F, Ferlay J, et al (2005). Global cancer statistics. Cancer J Clin, 55, 74- 108.

Pasquini M and Biondi M (2007). Depression in cancer patients: a critical review. Clini Practice Epidemiol Mental Health, $\mathbf{5}, 502-11$.

William M (2002). Is it appropriate to screen palliative care patients for depression? American J Hospice Palliative Care, 2, 112-4.

Zabalegui A, Sanchez S, Sanchez D, et al (2005). Nursing and cancer support groups. J Advance Nurs, 4, 369-81. 